\title{
Effect of docosahexaenoic acid in a mouse facial nerve injury model
}

Milan Makwana ${ }^{1}$

Simon Dyall ${ }^{2}$

Gennadij Raivich ${ }^{1}$

Adina Michael-Titus²

\section{Perinatal Brain Repair Group,}

Department of Obstetrics and Gynaecology and Department of Anatomy,

University College,

London

2. Neuroscience Centre,

Institute of Cell \& Molecular Sciences,

Bart's and the London School of Medicine and Dentistry,

Queen Mary University of London,

4, Newark Street,

London E1 2AT

We have previously reported in a model of spinal cord injury that the omega-3 polyunsaturated fatty acid docosahexaenoic acid (DHA) has significant neuroprotective effects after trauma in the central nervous system [1]. In the present study we extend our observations on the neuroprotective and neuroregenerative effects of DHA to a peripheral nerve injury model. Facial nerve transection in mice is a well-established model used in studies on axonal regeneration [2]. We carried out this nerve lesion in mice and we studied the effects of oral supplementation with DHA after injury, on neuronal and glial markers of injury, and on neurological outcome.

Female adult C57Bl6 mice (6 animals per group) were used throughout. Animals were placed for 1 week before surgery on a standard powdered diet. For the surgery, the right facial nerve, including the retroauricular branch, was transected at the styloid foramen under deep anaesthesia. After injury, mice continued to receive either the standard diet or a diet enriched with DHA $(300 \mathrm{mg} / \mathrm{kg} /$ day), for 14 days. On the last day of treatment, the neurological outcome was assessed by measuring the movement of the whiskers, on a scale from 0 (no movement) to 3 (normal movement) [3]. Then the animals were killed and perfused intracardially with $4 \%$ paraformaldehyde. The brainstem was removed, post-fixed and cryoprotected. $20 \mu \mathrm{m}$ sections were cut at the level of the facial nucleus, and were incubated with primary antisera for calcitonin gene-related peptide (CGRP, 1:400, peninsula) and galanin (1:400, Peninsula), as neuronal markers, and for the integrin subu-

nits $\alpha \mathrm{M}$ (1:5000, Pharmingen) and $\beta 1$ (1:3000, Pharmingen), whose expression is microglial only, and mixed neuronal/microglial, respectively.

Total immunoreactivity for each antigen was quantified in 2 sections per animal. Data was expressed as means \pm SEM and analysed statistically using Student's $t$ test.

In the DHA-treated group there was a significant increase in the number of both CGRP- and galanin-immunoreactive sprouts, vs. the lesioned control animals kept on the normal diet $(+140 \%$ and $+90 \%$, respectively, both $p<0.05)$. A significant increase was also seen in integrin immunoreactivity, with an increase of $20 \%$ for the $\alpha \mathrm{M}$-, and $167 \%$ for the $\beta 1$-integrin subunit, respectively (both $\mathrm{p}<0.05$ ) in animals receiving the DHA-supplemented diet, in comparison with animals on the control diet. The intake of DHA after injury also led to a better functional outcome, although the increase in whisker mobility 14 days after injury (+ $67 \%$ vs. controls) was not statistically significant.

These results show that the dietary supplementation with DHA in the period following facial nerve injury has significant effects on the response of both neurons and microglia to injury, and appears to promote a pro-regenerative response. This suggests that DHA could have a neuroprotective potential in the treatment of peripheral nerve injury.

\section{References}

1. King VR, et al. J Neurosci 2006; 26: 4672-80.

2. Moran LB, Graeber MB. Brain Res 2004; 44: 154-78.

3. Raivich G, et al. Neuron 2004; 43: 57-67.

POSTERS 